\title{
Evaluation of the Effects of Mouthwash on the Morphology and Cell Viability of Osteoblast-Like Cells
}

\author{
JaeHyung Lim $\mathbb{D},{ }^{1}$ Ji Eun Lee $\mathbb{D},{ }^{2}$ Chang-Joo Park $\mathbb{D},{ }^{3}$ and Jun-Beom Park $\mathbb{D}^{2}$ \\ ${ }^{1}$ Division of Oral and Maxillofacial Surgery, Department of Dentistry, Korea University Ansan Hospital, \\ Ansan 15355, Republic of Korea \\ ${ }^{2}$ Department of Periodontics, College of Medicine, The Catholic University of Korea, Seoul 06591, Republic of Korea \\ ${ }^{3}$ Division of Oral \& Maxillofacial Surgery, Department of Dentistry, Hanyang University College of Medicine, \\ Seoul 04763, Republic of Korea \\ Correspondence should be addressed to Jun-Beom Park; jbassoonis@yahoo.co.kr
}

Received 11 November 2021; Revised 16 January 2022; Accepted 26 January 2022; Published 4 February 2022

Academic Editor: Jozsef Szalma

Copyright (C) 2022 JaeHyung Lim et al. This is an open access article distributed under the Creative Commons Attribution License, which permits unrestricted use, distribution, and reproduction in any medium, provided the original work is properly cited.

\begin{abstract}
This study evaluated the effects of multiple mouthwashes on the cellular viability or the morphology of preosteoblasts. Mouse calvarial osteoblast-like cells were cultured and treated with mouthwashes of (1) benzydamine hydrochloride; (2) cetylpyridinium chloride and benzalkonium chloride; (3) methyl salicylate, menthol, eucalyptol, and thymol; and (4) sodium fluoride, xylitol, and chitosan. The treatment times were 30 seconds, 90 seconds, and 270 seconds. Cell morphology was evaluated with a microscope, and the viability of the treated cells was analyzed quantitatively using a commercially available kit. The untreated control group exhibited well-stretched fibroblast-like morphology. Treatment with mouthwash resulted in morphological changes in all groups. Treatment with sodium fluoride resulted in more noticeable changes. Treatment with mouthwash for 30 seconds produced a significant decrease in cell viability. An increase in time to 90 and 270 seconds did not produce additional noticeable changes. To conclude, commercially available mouthwashes created changes in cell morphology and decreased the cell viability of osteoblast-like cells irrespective of ingredients and treatment time.
\end{abstract}

\section{Introduction}

Mouthwash is used frequently in daily life and has the advantage of reaching areas that are not easily accessible with a toothbrush $[1,2]$. The use of mouthwash can aid people with daily oral hygiene [3]. Mouthwash has been used to heal soft tissue, reduce gingivitis, control plaque, reduce dental caries, control bad breath, and whiten teeth $[4,5]$. Moreover, chemical plaque control is the most commonly recommended means of oral hygiene after periodontal surgery [6]. A variety of mouthwashes is available by prescription or over the counter [7].

There are several main active components for mouthwashes including cetylpyridinium chloride, sodium fluoride, and essential oils [8]. Benzydamine has been recommended for prophylaxis of oral mucositis in head and neck cancers [9]. Cetylpyridinium chloride is a quaternary ammonium compound and has been used to reduce dental plaque and gingivitis [10]. Sodium fluoride has been reported to have an anticarious effect, and the use of mouthwash containing sodium fluoride can enhance remineralization of teeth [11]. Mouthwash containing methyl salicylate, menthol, eucalyptol, and thymol has been used as an adjunct to daily oral hygiene care due to the antiplaque and antigingivitis effects [12]. Previous reports have studied the possibilities of cytotoxic effects of mouthwash on cells $[6,13-15]$. This study examined the effects of multiple mouthwashes on the viability or morphology of osteoblast-like cells.

\section{Materials and Methods}

2.1. Cell Culture. Mouse calvarial osteoblast-like cells (MC3T3-E1) were deposited in 96-well plates at a density of $6.25 \times 10^{3}$ cells/well and maintained in $\alpha$-minimum 
essential medium ( $\alpha$ MEM, Welgene, Daegu, Korea) supplemented with $10 \%$ fetal bovine serum (Thermo Scientific, Logan, UT, USA), penicillin $100 \mathrm{U} / \mathrm{mL}$, and streptomycin $100 \mu \mathrm{g} / \mathrm{mL}$ (Gibco, Invitrogen, Carlsbad, CA, USA). The cultures were kept in a humidified atmosphere with $5 \% \mathrm{CO}_{2}$ and $95 \%$ air at $37^{\circ} \mathrm{C}$.

2.2. Evaluation of Cell Morphology after Use of Mouthwash. Figure 1 shows the overview of the study design. Six mouthwashes were tested in this study: (1) benzydamine hydrochloride $(150 \mathrm{mg} / 100 \mathrm{~mL}$; Tantum, Sama Pharm Co. Ltd., Wonjusi, Gangwon-do, Korea); (2) cetylpyridinium chloride (50 mg/ $100 \mathrm{~mL}$ ) and benzalkonium chloride (GUM, Sunstar Inc., Osaka, Japan); (3) methyl salicylate, L-menthol, eucalyptol, and thymol (IP, 3M, St. Paul, MN, USA); (4) sodium fluoride $(0.2 \mathrm{mg} / 1 \mathrm{~mL})$, xylitol, and chitosan (Cool Spearmint, 3M); and (5) sodium fluoride, xylitol, and chitosan (Mild Muscat, $3 \mathrm{M})$. The treatment times were 30 seconds, 90 seconds, and 270 seconds. An untreated culture sample served as the control. The morphological changes were observed under an inverted microscope (Leica DM IRM, Leica Microsystems, Wetzlar, Germany) after each treatment.

2.3. Quantitative Determination of Cell Viability. The cell viability of the osteoblast-like cells was analyzed quantitatively by a Cell Counting Kit-8 (Dojindo Molecular Technologies Inc., Rockville, MD). A water-soluble tetrazolium salt- 8 solution was added to the culture and incubated for four hours. The amount of generated formazan was analyzed as absorbance at a $450 \mathrm{~nm}$ wavelength using a microplate spectrophotometer system (BioTek, Winooski, VT).

2.4. Statistical Analysis. The results were presented as the mean \pm standard error of the mean of the experiments. A test of normality and the equality of variances in the samples were conducted. Two-way analysis of variance was used for evaluation of the effects of application time and types of gargles using a commercially available program (SPSS 12 for Windows, SPSS Inc., Chicago, IL, USA) with a level of significance at 0.05 .

\section{Results}

3.1. Evaluation of Cell Morphology and Cell Viability. In the microscopic evaluations, the untreated cells attached to the culture plate exhibited well-organized fibroblast-like actin cytoskeletons. Treatment of the osteoblast-like cells with Tantum resulted in an alteration in morphology (Figure 2). Treatment for longer times resulted in a more rounded shape. Similar trends were achieved in the GUM group. Alterations in cytoskeletal organization and progressive detachment from the culture plate were observed with longer treatment time (Figure 2). The relative cell viability was $16.6 \% \pm 1.2 \%, 17.1 \% \pm 1.8 \%$, and $17.6 \% \pm 0.4 \%$ for Tantum at 30, 90, and 270 seconds, respectively, when the untreated control was considered 100\% (100.0\% $\pm 19.5 \%)$ (Figure 3). The mean cell viability for the GUM group was $19.8 \% \pm 1.2 \%, 48.2 \% \pm 2.9 \%$, and $24.3 \% \pm 7.2 \%$ at 30,90 , and 270 seconds, respectively.
Cellular morphology after treatment with $3 \mathrm{M}$ mouthwashes is provided in Figure 4. Alterations in cytoskeletal organization were seen irrespective of the formulations. Agglomeration and detachment of the cells from the culture plate were noted.

The relative cell viability for IP was $16.9 \% \pm 0.8 \%$, $18.6 \% \pm 1.5 \%$, and $18.2 \% \pm 1.6 \%$ at 30,90 , and 270 seconds, respectively, when the untreated control was considered $100 \%$ (Figure 5). The mean cell viability for the Cool Spearmint group was $18.7 \% \pm 0.3 \%, 18.8 \% \pm 0.7 \%$, and $23.8 \% \pm$ $4.3 \%$ at 30,90 , and 270 seconds, respectively. The cell viability for the Mild Muscat group was $21.5 \% \pm 2.5 \%, 18.4 \% \pm$ $0.7 \%$, and $20.7 \% \pm 6.0 \%$ at 30,90 , and 270 seconds, respectively.

\section{Discussion}

This study showed that treatment with mouthwashes resulted in morphological changes and reduction in cell viability in all groups.

The cytotoxic effects of mouthwashes have been previously reported $[6,13]$. A previous in vitro study showed that undiluted mouthwashes induced near-complete cell death of human gingival and periodontal ligament fibroblasts 24 hours after only a 60-second treatment [6]. Dilutions of $15 \%$ to $20 \%$ for both essential oil mouthwashes resulted in reduction of cell death to $50 \%$, and dilutions of $10 \%$ to $15 \%$ of essential oils did not reduce cell migration [6]. A previous report tested various antiseptic agents on in vitro human gingival fibroblast proliferation [13]. The remaining viable cell density after application of $0.2 \%$ chlorhexidine was $35.2 \%$, and $0.15 \%$ benzydamine hydrochloride exhibited weaker cytotoxic effects, with the lowest cytotoxic effect in the essential oil group [13]. This present study showed that treatment with mouthwash for 30 seconds resulted in $20 \%$ to $30 \%$ cell viability.

Chemical plaque control is the most commonly recommended means of oral hygiene after periodontal surgery [6]. However, mouthwashes should not be considered a substitute for daily brushing and flossing [16, 17]. Swallowing or ingesting mouthwash should be avoided whenever possible [18] and can cause vomiting, nausea, or intoxication [19]. Children, especially young children, should not use mouthwash unless required or prescribed by a dental professional [20]. Manufacturers recommend specific durations or sequences of use depending on concentration and ingredients [21]. It is a matter of personal preference whether to rinse before or after brushing [22]. Toothpaste ingredients such as calcium hydroxide or aluminum hydroxide can form complexes with fluoride ions, reducing the effectiveness of mouthwashes $[23,24]$. If these ingredients are present in the toothpaste, it is recommended to rinse vigorously with water before using the mouthwash [25]. The use of diluted mouthwash can be considered because dilution of mouthwash including essential oils retained most of the antibacterial effects with minimal detrimental effects on human gingival and periodontal ligament fibroblasts [6].

Increasing evidence suggests that acetaldehyde, the first and genotoxic metabolite of ethanol, mediates the 


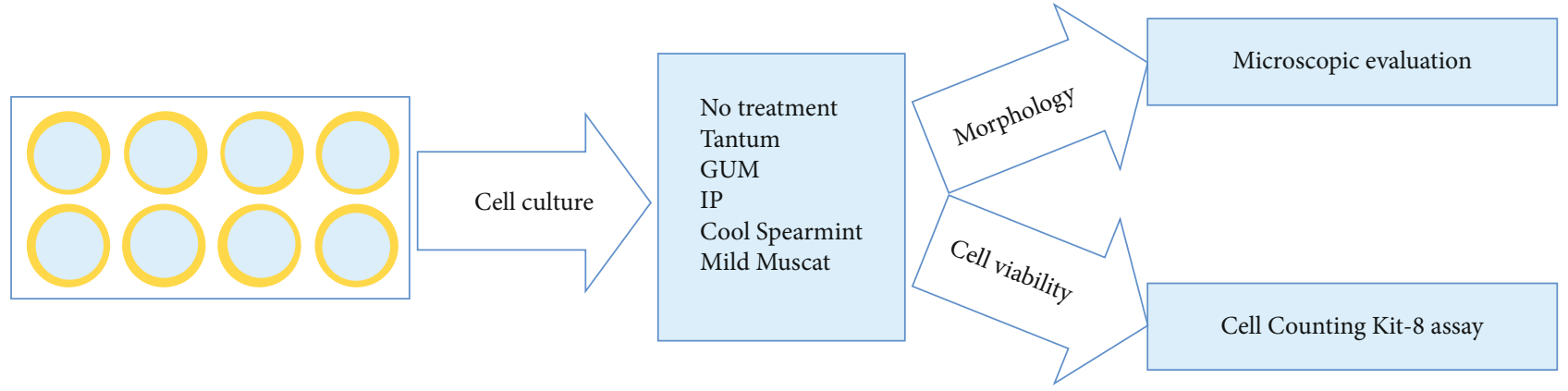

Figure 1: Diagram showing the overview of the study design.
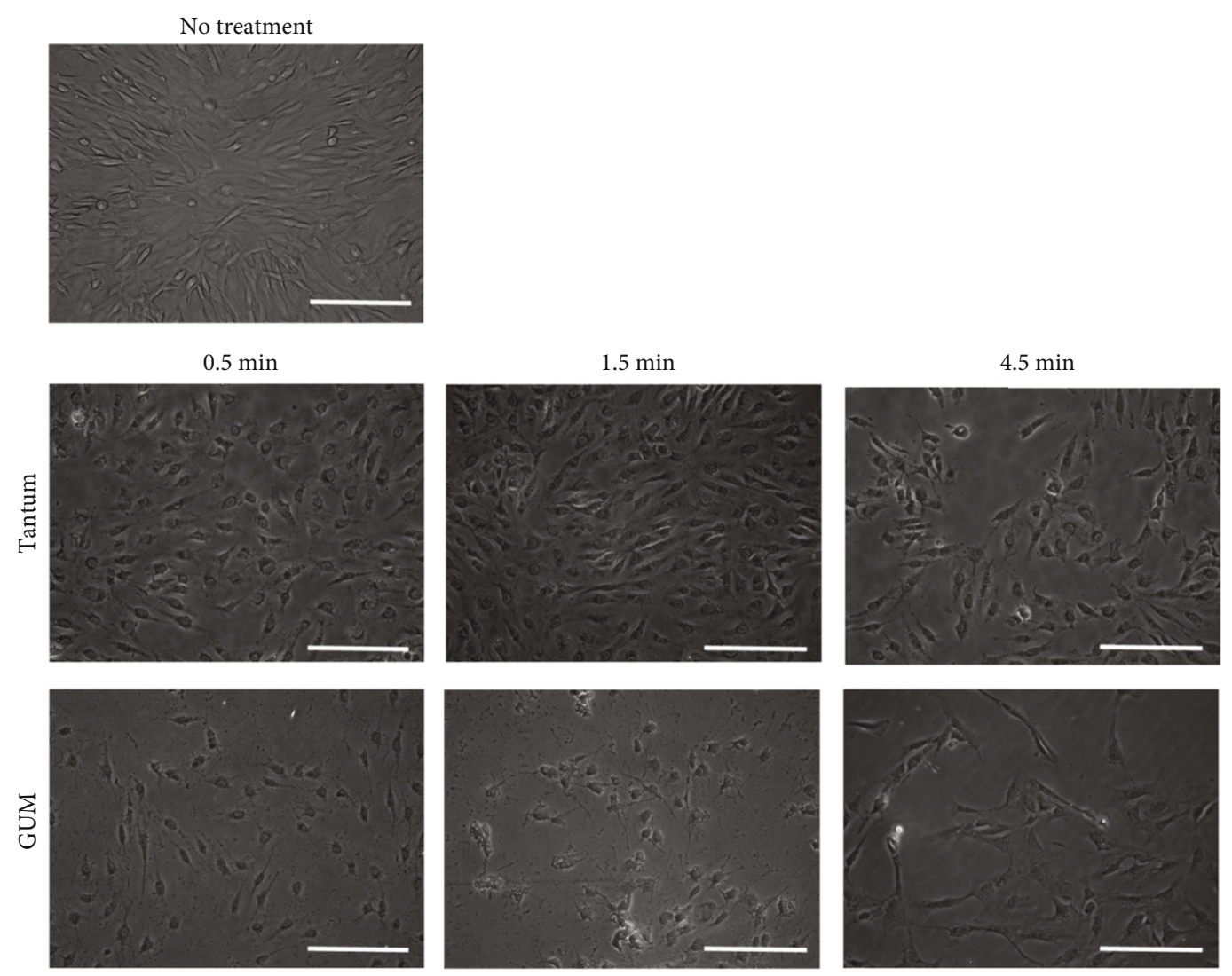

FIGURE 2: Evaluation of cellular morphology after treatment with benzydamine hydrochloride and cetylpyridinium chloride. The scale bar indicates $200 \mu \mathrm{m}$.

carcinogenicity of alcoholic beverages [26]. Ethanol is contained in a number of ready-to-use mouthwashes, typically between $5 \%$ and $27 \%$ volume [26]. The doses and administration times of antiseptics should be controlled carefully during dental application [13]. Further studies are required to determine the optimal application time and concentration of this antimicrobial agent to maximize reduction of the bacterial load and minimize cytotoxicity to the surrounding cells $[15,27]$.

According to previous publications on implants, the maintenance/management of implants is becoming more important than topics related to implant placement or osseointegration [28]. Among these subjects, especially in relation to peri-implantitis, it is essential to reduce bacteria and inflammation while delaying and preventing bone resorption/destruction [29]. In relation to the treatment of medication-related osteonecrosis of the jaw, which has been a major issue in dentistry for over 10 years, oral antimicrobial rinse cannot be left out $[30,31]$. Even now, it is a reality that systemic antibiotics, mechanical cleaning and/or removal, and local chemical rinsing are recommended for treatment of these bone-related lesions [32]. With regard to chemical rinse, in order to preserve vital bone as much as possible and reduce infection/inflammation, it is necessary to study the correct concentration and application time to reduce bacteria/inflammation while properly maintaining 


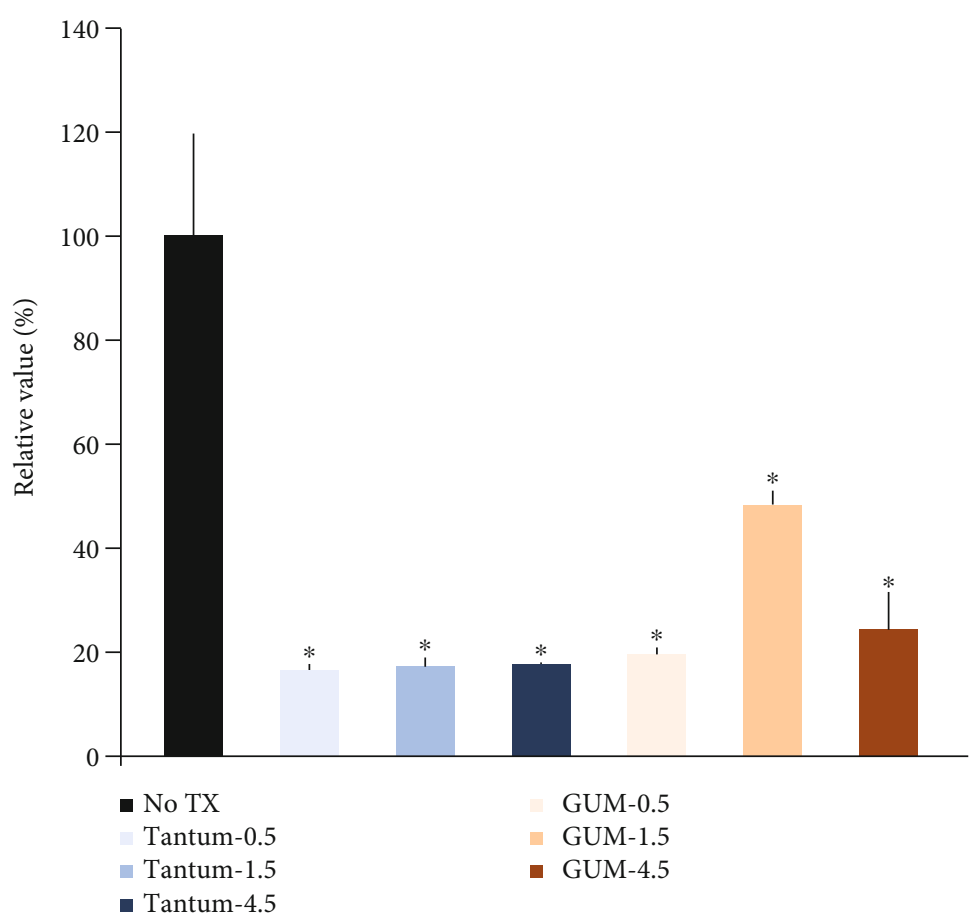

FIGURE 3: Cellular viability using Cell Counting Kit-8 after treatment with benzydamine hydrochloride and cetylpyridinium chloride.
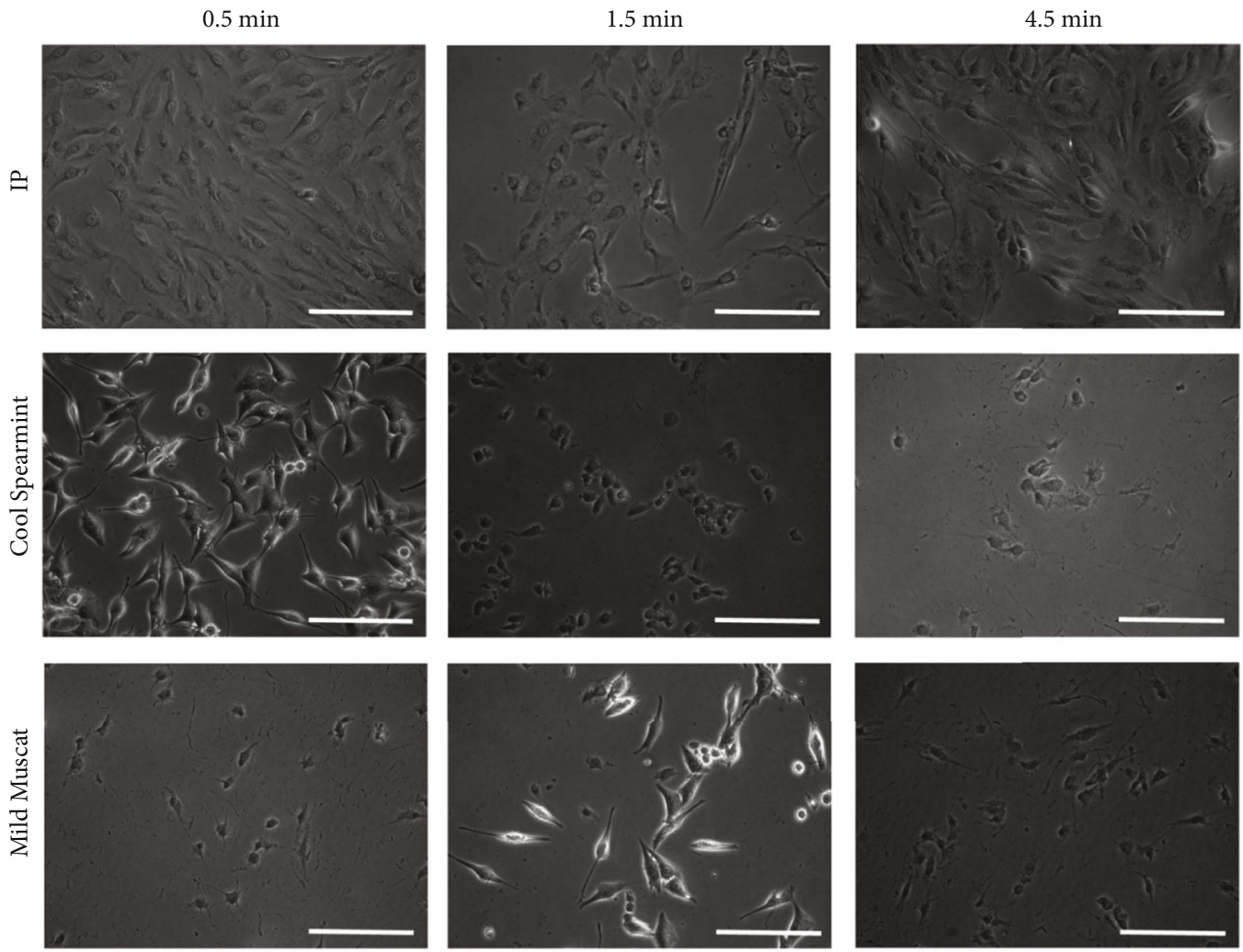

Figure 4: Evaluation of cellular morphology after treatment. The scale bar indicates $200 \mu \mathrm{m}$.

and restoring osteoblast activity. In relation to periimplantitis, it is essential to reduce bacteria and inflammation while delaying and preventing bone resorption/ destruction $[33,34]$. In addition, in relation to the treatment of medication-related osteonecrosis of jaw, oral antimicrobial rinse cannot be omitted [35]. In the future, 


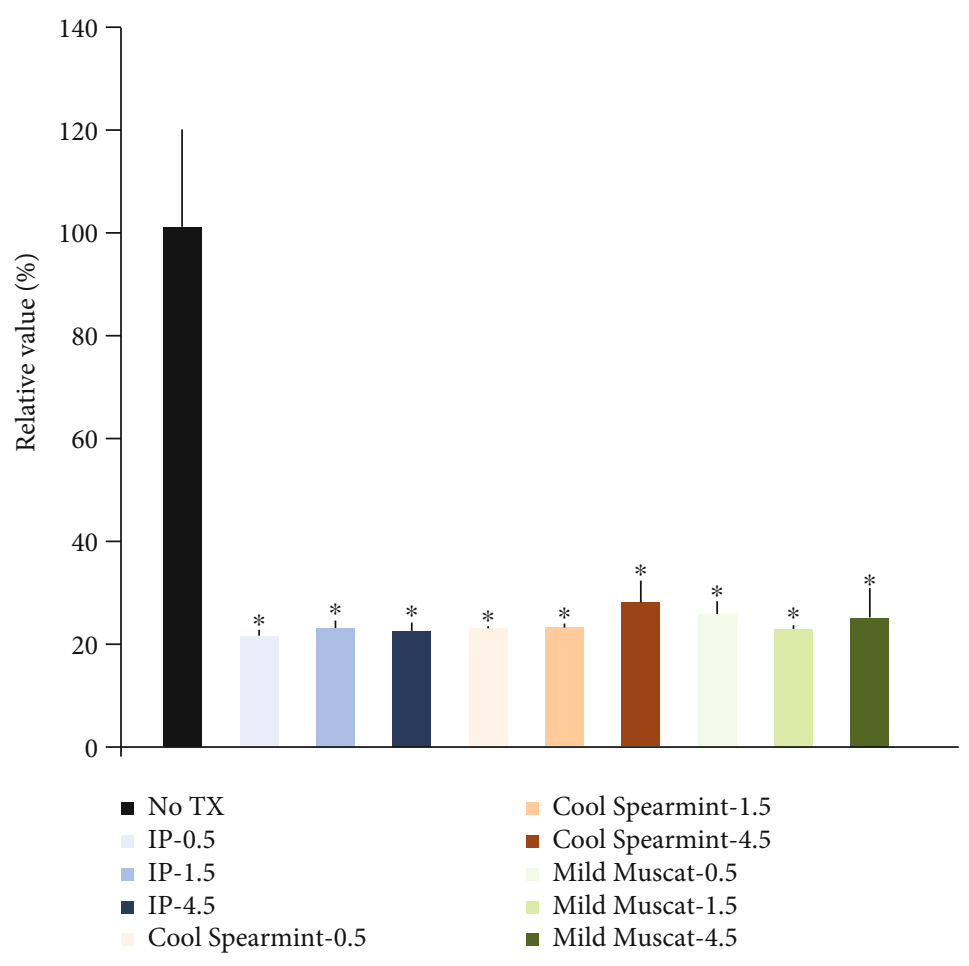

Figure 5: Cellular viability using the Cell Counting Kit-8 after treatment with mouthwashes containing methyl salicylate, L-menthol, eucalyptol, and thymol or sodium fluoride, xylitol, and chitosan.

similar experiments or in vivo experiments can be conducted to compare with these results and this result may serve as a baseline.

\section{Conclusions}

This study examined the effects of mouthwashes on the viability or morphology of osteoblast-like cells. The results showed that treatment with mouthwashes resulted in morphological changes and reduction in cell viability in all groups, with more noticeable changes by sodium fluoride. Collectively, commercially available mouthwashes resulted in changes in cell morphology and decreased cell viability of osteoblast-like cells irrespective of ingredients and treatment time.

\section{Data Availability}

All data generated or analyzed during this study are included in the published article.

\section{Conflicts of Interest}

The authors confirm that they have no competing interests.

\section{Authors' Contributions}

JaeHyung Lim and Ji Eun Lee contributed equally.

\section{Acknowledgments}

This research was supported by the National Research Foundation of Korea (NRF) grant funded by the Korea government (MSIT) (No. 2020R1A2C4001624). This study was also supported by Research Fund of Seoul St. Mary's Hospital, The Catholic University of Korea.

\section{References}

[1] F. A. Alshehri, "The use of mouthwash containing essential oils (LISTERINE ${ }^{\circledR}$ ) to improve oral health: a systematic review," Saudi Dental Journal, vol. 30, no. 1, pp. 2-6, 2018.

[2] I. Akwagyiram, A. Butler, R. Maclure, P. Colgan, N. Yan, and M. L. Bosma, "A randomised clinical trial to evaluate the effect of a $67 \%$ sodium bicarbonate-containing dentifrice on $0.2 \%$ chlorhexidine digluconate mouthwash tooth staining," $B M C$ Oral Health, vol. 16, no. 1, p. 79, 2016.

[3] H. M. Choi, K. Han, Y. G. Park, and J. B. Park, "Associations among oral hygiene behavior and hypertension prevalence and control: the 2008 to 2010 Korea National Health and Nutrition Examination Survey," Journal of Periodontology, vol. 86, no. 7, pp. 866-873, 2015.

[4] Z. L. S. Brookes, R. Bescos, L. A. Belfield, K. Ali, and A. Roberts, "Current uses of chlorhexidine for management of oral disease: a narrative review," Journal of Dentistry, vol. 103, article 103497, 2020.

[5] P. Ntovas, K. Masouras, and P. Lagouvardos, "Efficacy of nonhydrogen peroxide mouthrinses on tooth whitening: an in vitro study," ournal of Esthetic and Restorative Dentistry, vol. 33, no. 7, pp. 1059-1065, 2021.

[6] I. Tsourounakis, A. A. Palaiologou-Gallis, D. Stoute, P. Maney, and T. E. Lallier, "Effect of essential oil and chlorhexidine mouthwashes on gingival fibroblast survival and migration," Journal of Periodontology, vol. 84, no. 8, pp. 1211-1220, 2013. 
[7] K. Joshipura, F. Muñoz-Torres, J. Fernández-Santiago, R. P. Patel, and A. Lopez-Candales, "Over-the-counter mouthwash use, nitric oxide and hypertension risk," Blood Pressure, vol. 29, no. 2, pp. 103-112, 2020.

[8] J. Latimer, J. L. Munday, K. M. Buzza, S. Forbes, P. K. Sreenivasan, and A. J. McBain, "Antibacterial and anti-biofilm activity of mouthrinses containing cetylpyridinium chloride and sodium fluoride," BMC Microbiology, vol. 15, no. 1, p. 169, 2015.

[9] M. Rastogi, R. Khurana, S. Revannasiddaiah et al., "Role of benzydamine hydrochloride in the prevention of oral mucositis in head and neck cancer patients treated with radiotherapy (>50 Gy) with or without chemotherapy," Support Care Cancer, vol. 25, no. 5, pp. 1439-1443, 2017.

[10] A. Vergara-Buenaventura and C. Castro-Ruiz, "Use of mouthwashes against COVID-19 in dentistry," The British Journal of Oral \& Maxillofacial Surgery, vol. 58, no. 8, pp. 924-927, 2020.

[11] S. Ali, I. Farooq, A. M. Al-Thobity, K. S. Al-Khalifa, K. Alhooshani, and S. Sauro, "An in-vitro evaluation of fluoride content and enamel remineralization potential of two toothpastes containing different bioactive glasses," Bio-medical Materials and Engineering, vol. 30, no. 5-6, pp. 487-496, 2020.

[12] D. Osso and N. Kanani, "Antiseptic mouth rinses: an update on comparative effectiveness, risks and recommendations," Journal of Dental Hygiene, vol. 87, no. 1, pp. 10-18, 2013.

[13] N. A. Yayli, S. K. Tunc, B. U. Degirmenci, A. Dikilitas, and M. Taspinar, "Comparative evaluation of the cytotoxic effects of different oral antiseptics: a primary culture study," Nigerian Journal of Clinical Practice, vol. 24, no. 3, pp. 313-320, 2021.

[14] I. S. Song, J. E. Lee, and J. B. Park, "The effects of various mouthwashes on osteoblast precursor cells," Open Life Sciences, vol. 14, no. 1, pp. 376-383, 2019.

[15] J. B. Park, G. Lee, B. G. Yun, C. H. Kim, and Y. Ko, “Comparative effects of chlorhexidine and essential oils containing mouth rinse on stem cells cultured on a titanium surface," Molecular Medicine Reports, vol. 9, no. 4, pp. 1249-1253, 2014.

[16] H. Raison, R. Corcoran, and R. V. Harris, "Is toothbrushing behaviour habitual? Cues, context, motivators and patient narratives," Community Dentistry and Oral Epidemiology, vol. 49, no. 5, pp. 478-486, 2021.

[17] M. H. Raison, R. Corcoran, G. Burnside, and R. Harris, "Oral hygiene behaviour automaticity: are toothbrushing and interdental cleaning habitual behaviours?," Journal of Dentistry, vol. 102, article 103470, 2020.

[18] D. W. Lachenmeier, Y. B. Monakhova, M. Markova, T. Kuballa, and J. Rehm, "What happens if people start drinking mouthwash as surrogate alcohol? A quantitative risk assessment," Food and Chemical Toxicology, vol. 51, pp. 173178, 2013.

[19] Y. Fuse, Y. Ito, M. Yamaguchi, and N. Tsukada, "High ingestion rate of iodine from povidone-iodine mouthwash," in Biological Trace Element Research, Springer, 2021.

[20] E. Kidd, "Caries control from cradle to grave," Dental Update, vol. 37, no. 10, pp. 651-656, 2010.

[21] S. Takenaka, T. Ohsumi, and Y. Noiri, "Evidence-based strategy for dental biofilms: current evidence of mouthwashes on dental biofilm and gingivitis," Japanese Dental Science Review, vol. 55, no. 1, pp. 33-40, 2019.

[22] E. Laing, P. Ashley, D. Gill, and F. Naini, "An update on oral hygiene products and techniques," Dental Update, vol. 35, no. 4, pp. $270-279,2008$.
[23] F. Lippert, "An introduction to toothpaste - its purpose, history and ingredients," Monographs in Oral Science, vol. 23, 2013.

[24] V. Y. Reshetnyak, O. V. Nesterova, O. I. Admakin et al., "Evaluation of free and total fluoride concentration in mouthwashes via measurement with ion-selective electrode," BMC Oral Health, vol. 19, no. 1, p. 251, 2019.

[25] A. Gupta and J. Gallagher, "Formulation and fluoride content of dentifrices: a review of current patterns," British Dental Journal, 2021.

[26] D. W. Lachenmeier, S. Gumbel-Mako, E. M. Sohnius, A. Keck-Wilhelm, E. Kratz, and G. Mildau, "Salivary acetaldehyde increase due to alcohol-containing mouthwash use: a risk factor for oral cancer," International Journal of Cancer, vol. 125 , no. 3, pp. 730-735, 2009.

[27] J. Y. Han, S. H. Park, J. Kim, K. G. Hwang, and C. J. Park, "Clinical factors affecting the longevity of fixed retainers and the influence of fixed retainers on periodontal health in periodontitis patients: a retrospective study," Journal of Periodontal \& Implant Science, vol. 51, no. 3, pp. 163-178, 2021.

[28] M. Gulati, V. Govila, V. Anand, and B. Anand, "Implant maintenance: a clinical update," International Scholarly Research Notices, vol. 2014, Article ID 908534, 8 pages, 2014.

[29] R. Smeets, A. Henningsen, O. Jung, M. Heiland, C. Hammächer, and J. M. Stein, "Definition, etiology, prevention and treatment of peri-implantitis-a review," Head \& Face Medicine, vol. 10, no. 1, 2014.

[30] N. A. AlDhalaan, A. BaQais, and A. Al-Omar, "Medicationrelated osteonecrosis of the jaw: a review," Cureus, vol. 12, no. 2, article e6944, 2020.

[31] P. G. C. Leite de Marcelos, D. Perez, D. M. Soares et al., "The effects of zoledronic acid on the progression of experimental periodontitis in rats: histological and microtomographic analyses," Journal of Periodontal \& Implant Science, vol. 51, no. 4, pp. 264-275, 2021.

[32] A. J. van Winkelhoff, "Antibiotics in the treatment of periimplantitis," The Journal of Oral Implantology, vol. 5, pp. S43-S50, 2012.

[33] A. Monje, A. Insua, and H. Wang, "Understanding periimplantitis as a plaque-associated and site-specific entity: on the local predisposing factors," Journal of Clinical Medicine, vol. 8, no. 2, p. $279,2019$.

[34] E. Sánchez-Fernández, A. Magán-Fernández, F. O'Valle, M. Bravo, and F. Mesa, "Hyaluronic acid reduces inflammation and crevicular fluid IL- $1 \beta$ concentrations in peri-implantitis: a randomized controlled clinical trial," Journal of Periodontal \& Implant Science, vol. 51, no. 1, pp. 63-74, 2021.

[35] L. He, X. Sun, Z. Liu, Y. Qiu, and Y. Niu, "Pathogenesis and multidisciplinary management of medication-related osteonecrosis of the jaw," International Journal of Oral Science, vol. 12, no. 1, p. $30,2020$. 